\title{
An Effective On-line Polymer Characterization Technique by Using SALS Image Processing Software and Wavelet Analysis
}

\author{
Guang-ming Xian, ${ }^{1}$ Jin-ping $Q u^{,}{ }^{2}$ and Bi-qing Zeng ${ }^{1}$ \\ ${ }^{1}$ Computer Engineering Department, South China Normal University, Foshan, Guangdong 528225, China \\ ${ }^{2}$ National Engineering Research Center of Novel Equipment for Polymer Processing, The Key Laboratory of Polymer \\ Processing Engineering, South China University of Technology, Ministry of Education, Guangzhou 510641, China
}

Correspondence should be addressed to Jin-ping Qu, xgm20011@126.com

Received 29 October 2008; Accepted 9 December 2008

Recommended by Peter Stockwell

\begin{abstract}
This paper describes an effective on-line polymer characterization technique by using small-angle light-scattering (SALS) image processing software and wavelet analysis. The phenomenon of small-angle light scattering has been applied to give information about transparent structures on morphology. Real-time visualization of various scattered light image and light intensity matrices is performed by the optical image real-time processing software for SALS. The software can measure the signal intensity of light scattering images, draw the frequency-intensity curves and the amplitude-intensity curves to indicate the variation of the intensity of scattered light in different processing conditions, and estimate the parameters. The current study utilizes a one-dimensional wavelet to delete noise from the original SALS signal and estimate the variation trend of maximum intensity area of the scattered light. So, the system brought the qualitative analysis of the structural information of transparent film success.
\end{abstract}

Copyright (C) 2008 Guang-ming Xian et al. This is an open access article distributed under the Creative Commons Attribution License, which permits unrestricted use, distribution, and reproduction in any medium, provided the original work is properly cited.

\section{INTRODUCTION}

Small-angle light scattering (SALS) techniques offer a number of advantages for the investigation of the nature and behavior of polymer materials. Nonintrusive characterization of the flow field of transparent film is an essential step toward an implementation of a structural control system that can regulate the structure development during processing.

A combination of in situ birefringence and depolarized light-scattering experiments was used to study the formation of an ordered cylindrical microstructure in a polystyreneblock-polyisoprene copolymer melt under a shear flow field [1]. A new multivariable measurement approach [2] for characterizing and correlating the nanoscale and microscale morphology of crystal-amorphous polymer blends with melt-phase behavior is described. A vertical small-angle light scattering instrument optimized for examining the scattering and light transmitted from structures ranging from 0.5 to $50 \mu \mathrm{m}$, thereby spanning the size range characteristic of the initial-to-late stages of thermal-phase transitions (e.g., meltphase separation and crystallization) in crystal-amorphous polymer blends, was constructed. The present paper explores an effective means of characterizing structural changes of poly(vinyl chloride) (PVC) particles during gelation and fusion of PVC plastisols with small-angle light scattering. The SALS method was shown to provide an in situ observation of swelling of PVC particles as well as quantitative information of average size of swollen particles while they are in progress of gelation and fusion. In addition, the SALS method enabled one to evaluate the relative solvent power of plasticizers from the manner of increase in the correlation distances [3].

Recently, there has been increasing interest in understanding the complex processes that take place during the processing of polymer blends [4-6]. For this purpose, the small-angle light scattering technique is a very efficient method [7-9]. One of the important characteristics of light scattering is that it is a nondestructive test. This makes it possible to follow the time evolution of the phase separation process. The scattering pattern is a direct reflection of orientation, shape, and size of the structure. Another advantage is that with an appropriate choice of instruments, one can follow extremely fast events having a low optical contrast $[10,11]$. 
For optimum mechanical and optical properties, finestructured morphologies on a submicron scale are generally desired, as fine dispersions or cocontinuous morphologies with a low volume fraction of one component [12].

The light scattering method is valid for giving information about overall structures but is difficult to use for extracting local information on morphology. Recently, a digital image processing technique has shown its utility in analyzing the pattern formation in polymer systems [13]. Small angle light scattering study provides information on changes of morphology [14]. Various light scattering and optical techniques have been investigated as potential candidates for characterization of multiphase polymeric materials [15]. SALS is one of the tools that can be used to study a phase separation. It is shown that SALS can be used to discriminate between nucleation and growth (NG) and spinodal decomposition (SD) even when both give a pattern composed of a ring [16]. The gelation mode as a function of time was analyzed for polymers and polymer-carbon fiber composites by using polarized microscopy and polarized light scattering in terms of the formation of polymer spherulites [17]. Endoh et al. [18] aimed at elucidating the influence of shearinduced structures (shear-enhanced concentration fluctuations and/or shear-induced phase separation), as observed by rheo-optical methods with small-angle light scattering under shear flow (shear-SALS) and shear-microscopy, on viscoelastic properties in semidilute polystyrene (PS) solutions of $6.0 \mathrm{wt} \%$ concentration using dioctyl phthalate (DOP) as a $\Theta$ solvent and tricresyl phosphate (TCP) as a good solvent. Small-angle light scattering was used to determine the binary interaction parameter in a molten blend of linear polyethylene $(\mathrm{LPE})(\mathrm{Mw}=52 \mathrm{~kg} / \mathrm{mol}, \quad \mathrm{PDI}=2.9)$ and linear low-density polyethylenes (LLDPEs) based on homogeneous ethylene-1-butene copolymers (LLDPE1, $18.7 \mathrm{~mol} \%$ butane branches, $\mathrm{Mw}=58.1 \mathrm{~kg} / \mathrm{mol}$, and LLDPE-2, $5.9 \mathrm{~mol} \%$ butene branches, $\mathrm{Mw}=70 \mathrm{~kg} / \mathrm{mol}$ ). Our results are significant because they show that the low optical contrast between coexisting phases in polyolefin blends does not limit the determination of phase boundaries by SALS as was previously assumed. The blends studied exhibit upper critical solution temperature behavior [19].

The quantitative analysis software system can be integrated into the picture archiving and communication system [20]. The combination of advances in charge-coupleddevice (CCD) fabrication, camera design, digital interface technology, and software development has enabled scientific imaging device manufacturers to overcome the challenges created by the wide range of requirements [21]. The software kits, which include PCI device driver and image processing package, are developed based on Windows OS [22].

In our study, the transparent film is viewed through crossed polarizers to reveal the light scattering pattern. A high-speed CCD camera is used to record the SALS signal in real time with different process conditions for subsequent analysis. Modification algorithm has been proposed to eliminate the noise of multiple scattering. An optical image real-time analysis software has been developed for accurate modeling and simulation of the structural information of the transparent film. Visualization is performed via a highperformance analysis software which allows on-line data acquisition and processing the SALS signal. The experiments yield information regarding the trend of the maximum light intensity of the transparent film that can be compared under different processing conditions.

Wavelets have been used successfully in numerous applications ranging from analysis of flotation froth to countertops $[23,24]$. Lambert et al. aims at developing a more accurate measurement of the physical parameters of fractal dimension, and the size distribution of large fractal aggregates by small-angle light scattering. The theory of multiple scattering has been of particular interest in the case of fractal [25]. Ismail et al. outline how the wavelet transform, a hierarchical averaging scheme, can be used to perform both spatial and topological coarse-graining $n$ systems with multiscale physical behavior, such as Ising lattices and polymer models [26]. A brief description is given of a methodology that exploits guided ultrasonic waves, lasers and fiber optics, and simultaneous time-frequency analysis to interrogate the state of a material, component, or structure. The propagating ultrasound interrogates the host material in a manner providing a wealth of information when coupled with application of the Gabor wavelet transform to broadband dispersive waveforms. Recent results are presented pertaining to delamination detection within layered copper/polymer films [27].

Wavelet analysis (WA) is typically suited in applications where data contains both large and small scales of variation, such as small-angle light signal. We presented a new technique that can be used to analyze the structural information of transparent film on-line and nonintrusively while the material is processing. The technique is based on SALS, optical signal real-time analysis software, and wavelet transform method. It is shown that the proposed technique is easy to implement and provides more flexibility, approximating the relation between the intensity signal and the corresponding variation time. Applying this method to analyze structural information of transparent film will be of great interest, since it will contribute information on optical prosperities that have been proven to be useful for obtaining deep insights into the molecular and structural parameters of transparent film. In our experiments, the SALS signal denoised by wavelet analysis is better than the signal denoised by inducing $K_{f}$ factor. The variation trend of the SALS signal becomes clear, and the exceptional SALS signal can be accurately detected by wavelet decomposition [28].

In this paper, the results from a measurement technique are investigated. The method will be evaluated on the basis of light scattering measurements for a small range of scattering angles. These measurements have been taken with a fast CCD line scan camera and appropriate optics. An attempt is made to derive information from these measurements only. With the continuous wavelet transform, SALS image analysis methods are used to process the SALS signal. The purpose of the present work is to apply the optical image technique to characterize the structural informal of transparent film. In particular, we attempt to on-line the analysis of the light intensity signal. 


\section{THEORETICAL BACKGROUND FOR SALS}

When a light beam passes through a diffusion surface, the variation of propagation direction of the beam cannot be determined by the principle of geometrical optics because of scattering function of light beams on diffusion surface [29].

A transparent fluid is an optical phase object. In the experimental set-up for measuring flow fields in fluid flows by using speckle interferometry, the part of the arrangement for the object light beam is just like a subjective photographic system. Therefore, in general, speckle displacements are generated. The speckle displacements can change the intensity distribution of spatial speckle fields. As a result, the intensity distribution of a speckle interferogram is also changed. In this paper, the effect of variation of the intensity is analyzed and discussed in detail. Experimental results are shown. Methods for elimination of the multiple scattering effect are provided. This is advantageous to improve the quality of the speckle interferogram [30].

In our experiments, device performs real-time image analysis of the evolving light scattering signal. The experimental device incorporates an He-Ne laser generator, optics, a CCD camera, and a personal computer as its major hardware components. Software designed specifically for this application performs real-time analysis of the light scattering pattern. Intensities at various scattering and azimuthal angles are plotted at each time [31].

Figure 1 shows an experimental set-up for small-angle light-scattering measurement device. A laser light passes through polymer melts in the visual slit dies. A polarizer and an analyzer are placed before and after the polymer melts. The laser light first passes the polarizer, which removes one orthogonal component of the light [32]. The other component of light passes through the polymer melts with resulting scattering due to the orientation of molecular chain. The analyzer removes the second component since it is placed $90^{\circ}$ out of phase with respect to the analyzer. Therefore, any light that comes out of the analyzer is entirely due to the scattering within the polymer melts. The depolarized intensity of light that passes through the polarizer, polymer melts, and analyzer is recorded and related to the orientation of polymer melts. A CCD camera captures the image, and the total intensity of the image is determined in every 5 seconds. The total intensity is assumed proportional to orientation of molecular chain.

We assume that each column of the following matrix represents the intensities of one observed Raman spectrum at the selected wave shifts:

$$
D=\left[\begin{array}{cccc}
d_{1,1} & d_{1,2} & \cdots & d_{1, n} \\
d_{2,1} & d_{2,2} & \cdots & d_{2, n} \\
\cdots & \cdots & \cdots & \cdots \\
d_{m, 1} & d_{m, 2} & \cdots & d_{m, n}
\end{array}\right], \quad D \in R^{m x m}
$$

Therefore, each spectrum is represented by $(m)$ number of spectral intensities, and a total of $(n)$ spectrum exists. The dispersion matrix $Z$ [32] that represents the variation in the data is computed as

$$
Z=D^{T} D, \quad Z \in R^{m x m} .
$$

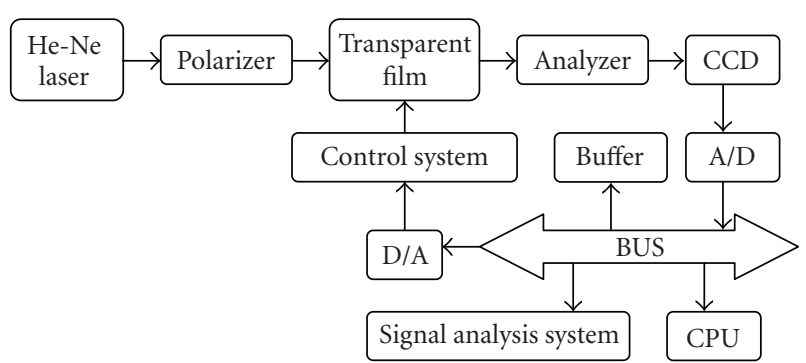

Figure 1: Experimental device for SALS.

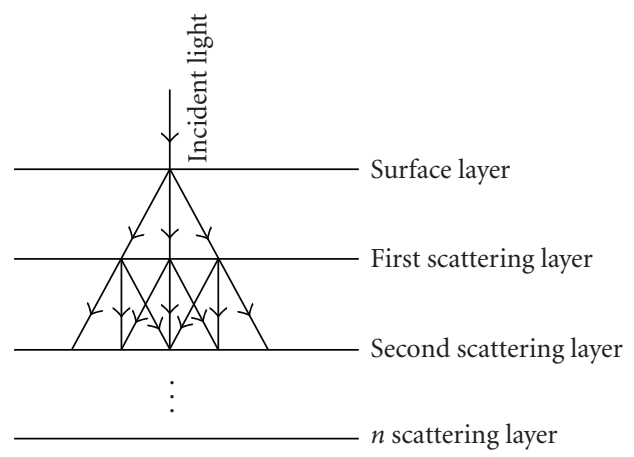

FIGURE 2: Diagram of multiple scattering.

The diagram of multiple scattering is shown in Figure 2. According to the effect of the sample on the incident light, the sample can be divided into a surface layer, a first scattering layer, a second scattering layer, and so forth. The incident light first impinges on the surface of the sample of the first scattering layer (random reflection) of the medium. The second one comes from the first scattering layer (because of the internal heterogeneity) and the third in turn [33].

The sketch of incidence beam with litter angle is shown in Figure 3. Here, $\alpha$ is the angle of incident light, and $d$ is the thickness of the sample.

Because of multiple light scattering caused by the thickness of the sample, the light scattering images will be dispersion and distortion models. A distortion model is constructed, and a correcting factor is introduced. Computer simulation is verified under some factual circumstance. Introducing the correcting factor improves the precision and the reliability of the image.

The measurable intensity of scattered light $I_{s}^{\prime}$ and the factual intensity of scattered light $I_{s}$ have the relationship

$$
I_{s}=K_{f} I_{s}^{\prime}
$$

where $K_{f}$ is the correcting factor that can be written as

$$
K_{f}=e^{(\tau d / \cos \varphi)} \tau d\left(\cos ^{-1} \varphi-1\right)\left\{e^{\left[\tau d\left(\cos ^{-1} \varphi-1\right)-1\right]}\right\}^{-1},
$$

where $\varphi$ is the scattering angle, and $\tau$ is the turbidity of the sample [34]. In a specific point, $\varphi$ is a constant.

Supposed $\tau$ (the turbidity of the sample) is the same, so correcting factor $K_{f}$ is only with relation to $d$ (the thickness of the transparent film). 


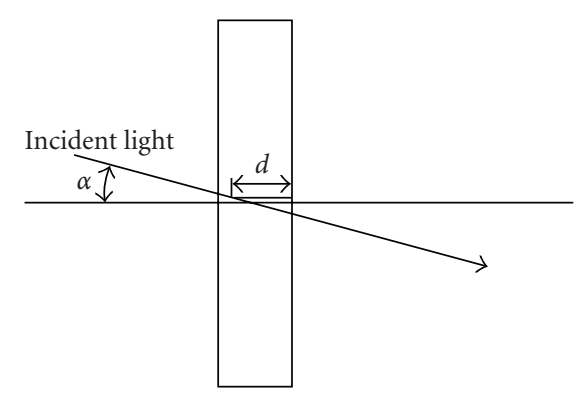

FIgURE 3: The sketch of incidence beam with litter angle.

\section{WAVELET ANALYSIS FOR MULTIPLE SCATTERING SALS SIGNAL}

Spectral analysis and time series methods are the most commonly used signal processing techniques. However, these methods were reported to provide a good solution only in the frequency domain and poor solution in the time domain. Like the Fourier transform (FT), the wavelet transform (WT) can be used to measure the frequency content of a signal. However, the WT differs from the FT in that it yields frequency information in a time-localized fashion $[35,36]$. This makes the WT far more effective than FT in identifying time-based phenomena.

Given a time varying signal $f(t)$, WTs consist of computed coefficients of inner products of the signal and a family of wavelets. In a continuous wavelet transform (CWT), the wavelet corresponding to scale $(a)$ and time location $(b)$ is

$$
\psi_{a, b}=\frac{1}{\sqrt{|a|}} \psi\left(\frac{t-b}{a}\right), \quad a, b \in R, a \neq 0,
$$

where $(a)$ and $(b)$ are the dilation and translation parameters, respectively. The CWT is defined as follows:

$$
\operatorname{CWT}\{x(t) ; a, b\}=\int x(t) \psi_{a, b}^{*}(t) d t,
$$

where $*$ denotes the complex conjugation. In this paper, Morlet wavelet function [37] was used, which can be represented as

$$
\psi(t)=e^{-t^{2} / 2} e^{j w_{0} t}
$$

Its CWT is

$$
\psi_{a, b}=\frac{1}{\sqrt{a}} e^{-1 / 2((t-b) / a)^{2}} e^{j w_{0}((t-b) / a)} .
$$

In $(8),(a)$ and $\left(w_{0}\right)$ can be changed, each way yields a different type of WT. The sample frequency of the wavelet function and the signal is $\left(f_{w}\right)$ and $\left(f_{s}\right)$, respectively; the relationship of the parameter $a$ and $w_{0}$ is

$$
a=\frac{w_{0} f_{s}}{2 \pi f_{w} f_{0}}
$$

where $\left(f_{0}\right)$ is the frequency focused signal energy.
When $a=2^{j}, b=k 2^{j}, j, k \in Z$, the WT becomes

$$
\psi_{j, k}=2^{-j / 2} \psi\left(2^{-j} t-k\right) .
$$

The discrete wavelet transform (DWT) is defined as

$$
c_{j, k}=\int f(t) \psi_{j, k}^{*}(t)
$$

where $\left(c_{j, k}\right)$ is a time frequency map of the original signal $f(t)$.

A multiresolution analysis approach is used in this work, in which

$$
\begin{aligned}
\phi_{j, k} & =2^{-j / 2} \phi\left(\frac{t-2^{j} k}{2^{j}}\right), \\
d_{j, k} & =\int f(t) \phi_{j, k}^{*}(t),
\end{aligned}
$$

where $\left(\phi_{j, k}\right)$ is a discrete scaling function, and $\left(d_{j, k}\right)$ is a scaling coefficient. When $j=0, \phi_{j, k}$ is the sampled version of the original signal. The DWT computes wavelet coefficients $c_{j, k}$ for $j=1, \ldots, J$, and scaling coefficients $d_{j, k}$ are given by

$$
\begin{aligned}
c_{j, k} & =\sum_{n} x[n] h_{j}\left[n-2^{j} k\right], \\
d_{j, k} & =\sum_{n} x[n] g_{j}\left[n-2^{j} k\right],
\end{aligned}
$$

where $x[n]$ are discrete time signals, $h_{j}\left[n-2^{j} k\right]$ are the discrete wavelets, the discrete equivalents to $2^{-j / 2} \psi\left(2^{-j}(t-\right.$ $\left.\left.2^{j} k\right)\right), g_{j}\left[n-2^{j} k\right]$ are called scaling sequence.

At each resolution $j>0$, the scaling coefficients and the wavelet coefficients are

$$
\begin{aligned}
c_{j+1, k} & =\sum_{n} g[n-2 k] d_{j, k}, \\
d_{j+1, k} & =\sum_{n} h[n-2 k] d_{j, k} .
\end{aligned}
$$

From a mathematical point of view, the structure of computations in a DWT is exactly an octave-band filter band [38]. The terms $(g)$ and $(h)$ are high-pass and low-pass filters derived from the analysis wavelet $\psi(t)$ and the scaling function $\phi(t)$. Hence, $c_{j, k}$ represents the high-frequency components of the signal $f(t)$ [39].

\section{EXPERIMENTAL CHARACTERIZATION FOR THE FLOW FIELD OF POLYMER MELTS}

\subsection{Experimental set-up}

\subsubsection{Transparent fluids used}

The results reported in this study were obtained with polystyrene (PS) and high-density polyethylene (HDPE). They are both transparent, which is necessary to perform visualization experiments. As they are commercial polymers that melt at high temperatures, they enabled the study to be performed under quasi-industrial conditions. 


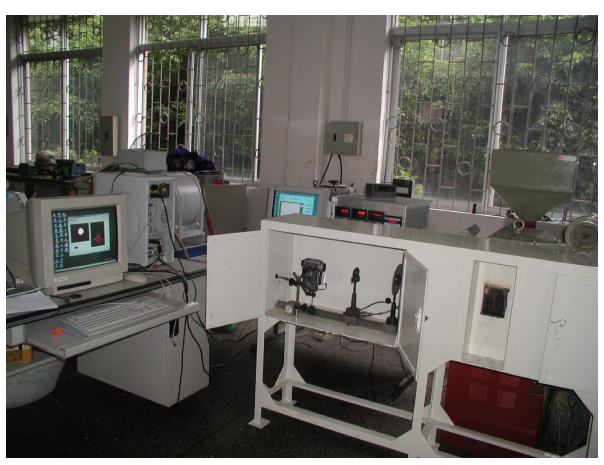

Figure 4: The experimental setup.

TABLE 1: Experimental conditions.

\begin{tabular}{cccc}
\hline Material & Rotate speed & Vibration amplitude & Vibration frequency \\
\hline \multirow{4}{*}{ PS HDPE } & $20 \mathrm{rpm}$ & $0.04 \mathrm{~mm}$ & $5 \mathrm{~Hz}$ \\
& $24 \mathrm{rpm}$ & $0.12 \mathrm{~mm}$ & $8 \mathrm{~Hz}$ \\
& $32 \mathrm{rpm}$ & $0.16 \mathrm{~mm}$ & $10 \mathrm{~Hz}$ \\
& & $0.20 \mathrm{~mm}$ & $12 \mathrm{~Hz}$ \\
\hline
\end{tabular}

\subsubsection{Optics}

An He-Ne laser is used as an incident light. Optical system and the polarization analyzer are detected by a CCD connected to a computer. Figure 4 shows the experimental set-up in our research.

\subsection{Experimental procedures}

Table 1 shows the experimental conditions. We used two kinds of material (PS and HDPE), three kinds of rotate speed (20 rpm, $24 \mathrm{rpm}$, and $32 \mathrm{rpm}$ ), five kinds of vibration amplitude $(0.04 \mathrm{~mm}, 0.08 \mathrm{~mm}, 0.12 \mathrm{~mm}, 0.16 \mathrm{~mm}$, and $0.20 \mathrm{~mm})$, and five kinds of vibration frequency $(5 \mathrm{~Hz}, 8 \mathrm{~Hz} 10 \mathrm{~Hz}$, $12 \mathrm{~Hz}$, and $15 \mathrm{~Hz}$ ). In the experiments, first the screw of the extruder rotated at constant speed. Second, we changed the amplitude and frequency of the screw, respectively. At the same time, a CCD camera captured the light scattering image of each processing condition. Finally, the optical image realtime analysis software characterized the flow field of polymer melts.

\subsection{Optical image real-time analysis system for SALS}

The optical image real-time processing software for SALS (see Figure 5), which provides a user-friendly interface already familiar to the users [40], is based on personal PC platform running under MS Windows operating system. Hardware specifics of A/D and digital I/O boards which are connected on PC motherboard impose some constraints and partly determine real-time software structure, especially disposition of its components at processors. The software is developed using Delphi7.0.

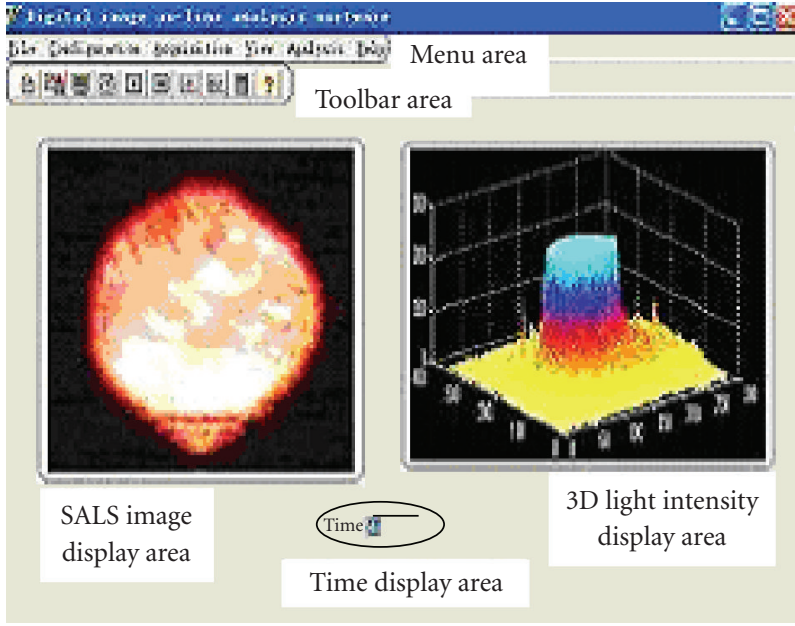

Figure 5: Optical image real-time processing software for SALS.

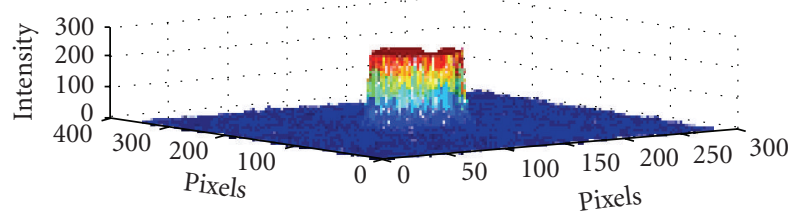

(a) $24 \mathrm{rpm}-0-0$

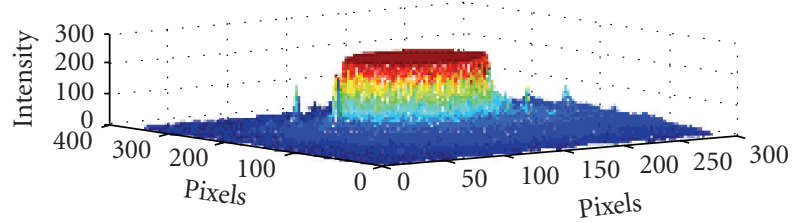

(b) $24 \mathrm{rpm}-10 \mathrm{~Hz}-0.20 \mathrm{~mm}$

FigURE 6: 3D light intensity compared images of HDPE at $24 \mathrm{rpm}$ screw rotate speed. (a) Without vibration, (b) with vibration frequency of $10 \mathrm{~Hz}$ and vibration amplitude of $0.20 \mathrm{~mm}$.

Real-time visualization of various scattered light image and light intensity matrices is performed by the host application. Algorithm of visualization starts with the selection of working parameters. The next step is setting parameters using corresponding dialog-boxes provided by the host application. Some of these parameters are vibration parameters (frequency, amplitude), display parameters (sizing grid, display scale, image translation, and rotate angle), and other parameters (rotate speed, material, sampling time). The light intensity matrix can be saved on disk of main workstation for further analyses.

The software can measure the signal intensity of light scattering images, draw the frequency-intensity curves and the amplitude-intensity curves to indicate the variation of the intensity of scattered light in different processing conditions, and estimate the hydrodynamic parameters. So, the system brought the qualitative analysis of the structural information of transparent film success [20]. 


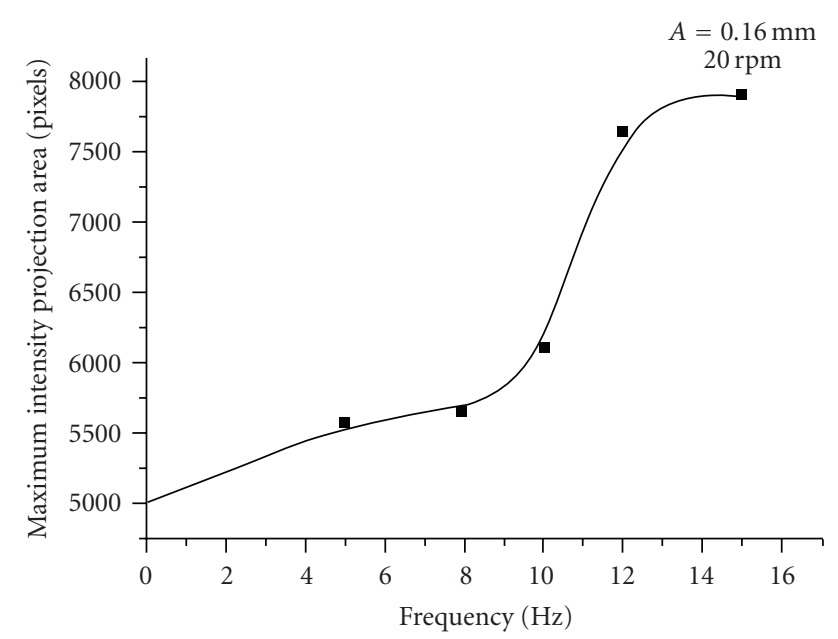

FIGURE 7: The relationship between maximum intensity projection area and vibration frequency of PS (rotate speed: $20 \mathrm{rpm}$, vibration amplitude: $0.16 \mathrm{~mm}$, and different vibration frequency).

Figure 6(a) shows a 3D light intensity image of HDPE at $24 \mathrm{rpm}$ screw rotate speed without vibration. Figure 6(b) shows light intensity image of HDPE at the same rotate speed with vibration frequency of $10 \mathrm{~Hz}$ and vibration amplitude of $0.20 \mathrm{~mm}$. In comparison with 3D light intensity image without vibration, 3D light intensity image with vibration has stronger light intensity. It is illustrated that the orientation of molecular chain increases because light intensity is proportional to orientation of molecular chain.

Figure 7 shows the variation trend of maximum intensity projection area with the increase of vibration frequency of PS at $20 \mathrm{rpm}$ rotate speed. As shown in Figure 8, with the increase of vibration frequency, the maximum intensity projection area becomes larger. It is because with the increase of vibration frequency, the molecular orientation of polymer melts also increases. As a consequence, the light intensity becomes stronger.

Figure 8 shows the relationship between maximum intensity projection area and vibration amplitude of HDPE at $24 \mathrm{rpm}$ screw rotate speed. From Figure 8, it is clear that with the increase of vibration amplitude, the maximum intensity projection area becomes larger. The molecular orientation of polymer melts increases is also the main reason of this optical phenomenon.

\subsection{SALS signal decomposition by wavelet analysis}

A signal including noise can be expressed as

$$
s(i)=f(i)+\sigma \cdot e(i), \quad i=0, \ldots, n-1,
$$

where $f(i)$ is the real signal, $e(i)$ is the noise, $\sigma$ is the coefficient of the noise, and $s(i)$ is the signal including noise.

The useful signal is included in the part of low frequency, and the noise is included in the part of high frequency. As shown in Figure 9, we used one-dimensional wavelet which decomposed the original signal into three level:

$$
S=\mathrm{Ca}_{3}+\mathrm{Cd}_{1}+\mathrm{Cd}_{2}+\mathrm{Cd}_{3},
$$

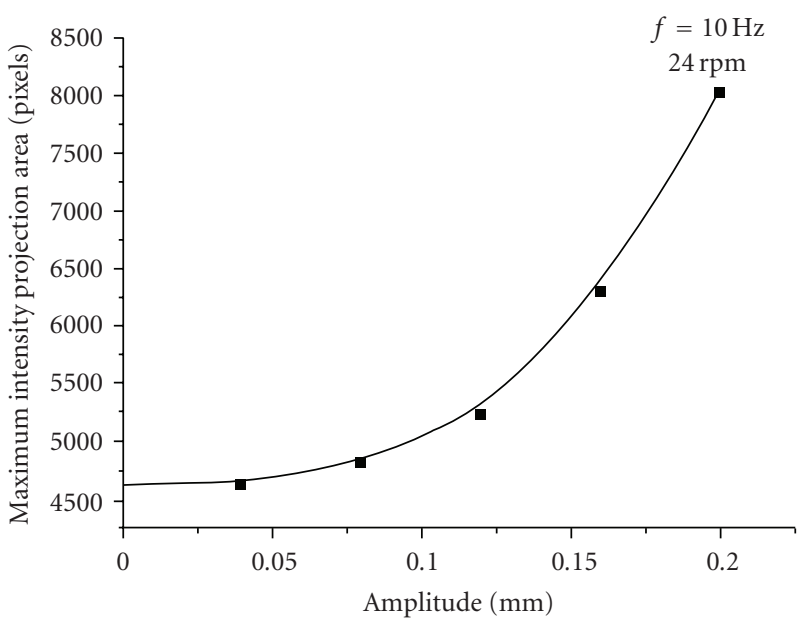

FIGURE 8: The relationship between maximum intensity projection area and vibration frequency of HDPE (rotate speed: $24 \mathrm{rpm}$, vibration frequency: $10 \mathrm{~Hz}$, and different vibration amplitude).

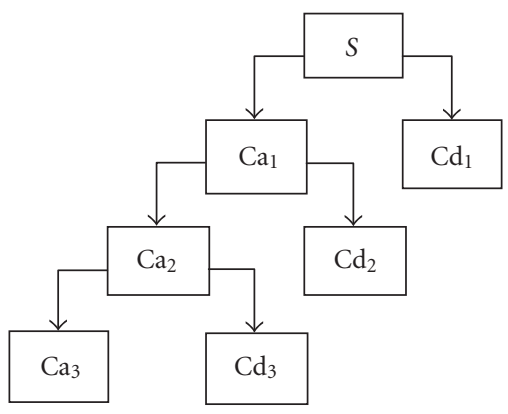

Figure 9: Sketch of multiresolution decomposition tree at level 3.

where $S$ is the original signal, $\mathrm{Ca}_{1}, \mathrm{Ca}_{2}, \mathrm{Ca}_{3}$ are the approximation coefficients of levels 1,2 , and 3 , and $\mathrm{Cd}_{1}, \mathrm{Cd}_{2}$, $\mathrm{Cd}_{3}$ are the detail coefficients of levels 1,2, and 3 .

\subsubsection{On-line SALS signal denosing by wavelet transform}

The performance of wavelet denoising is comparable to that of inducing $K_{f}$ correcting factor. Figure 10(a) is the normal SALS intensity signal. Figure 10(b) is the SALS signal with multiple scattering noise. Figure 10(c) shows the SALS signal denoising by $K_{f}$ factor. After being denoised by wavelet "sym6," the multiple scattering noise is eliminated and the signal (see Figure 10(e)) is becoming more smooth. From Figures 10(d) and 10(f), the residuals by wavelet decomposition are smaller than the residuals by inducing $K_{f}$ factor. Wavelet method is more successful in removing multiple scattering noise than that of inducing $K_{f}$ correcting factor.

\subsubsection{On-line wavelet analysis for the variation trend of the SALS signal}

The method of wavelet transform can reduce the ambiguities and accurately analyze the variation trend of the SALS 


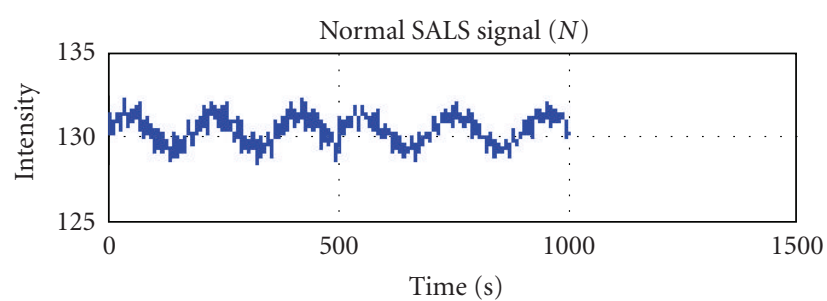

(a)

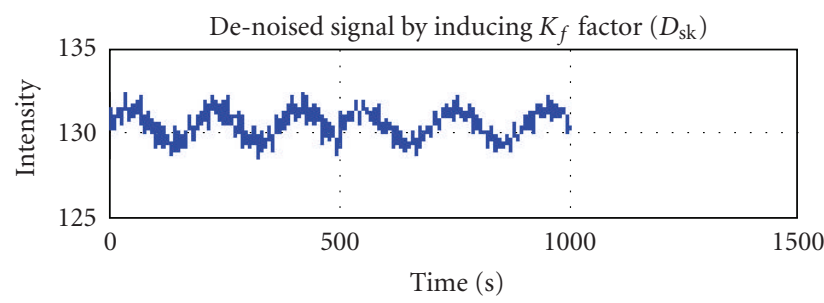

(c)

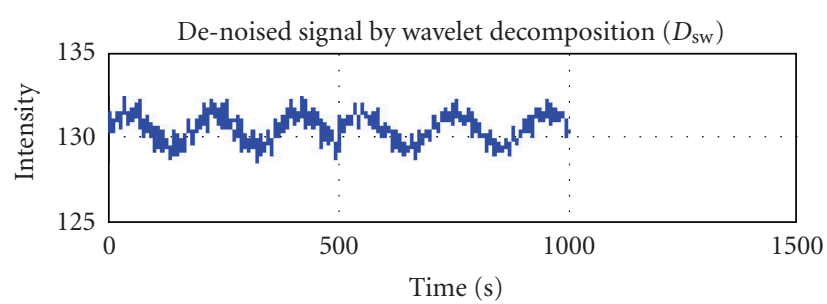

(e)

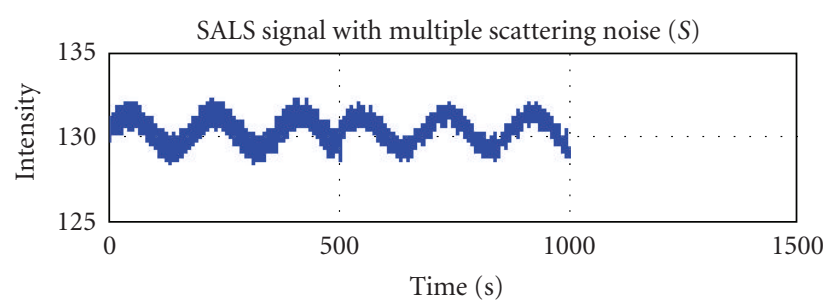

(b)

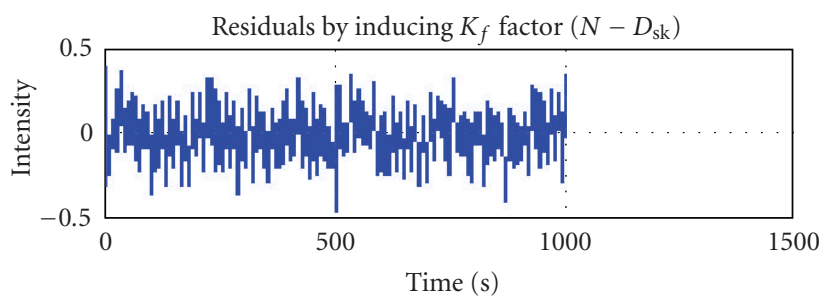

(d)

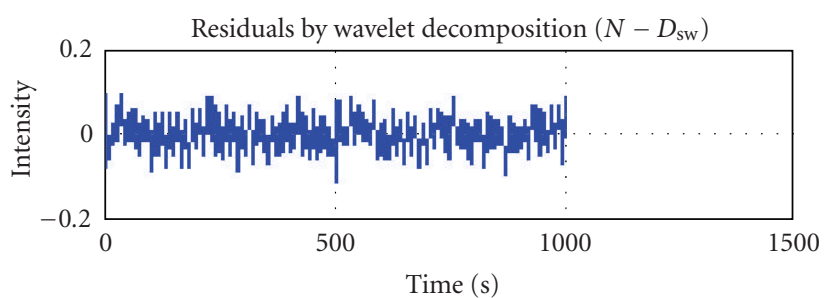

(f)

FIgURE 10: On-line SALS signal denosing by wavelet analysis (wavelet "sym6," level 3).

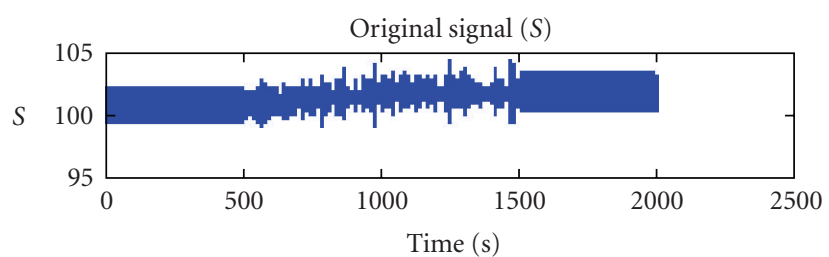

(a)

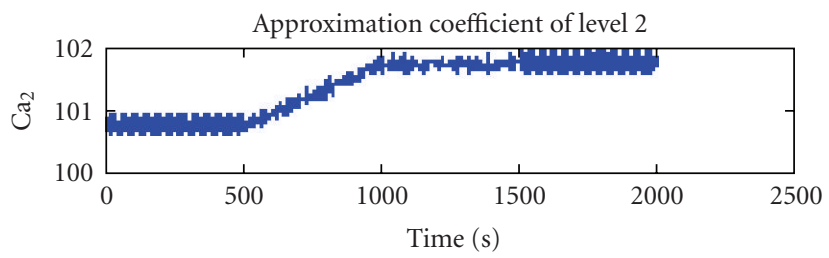

(c)

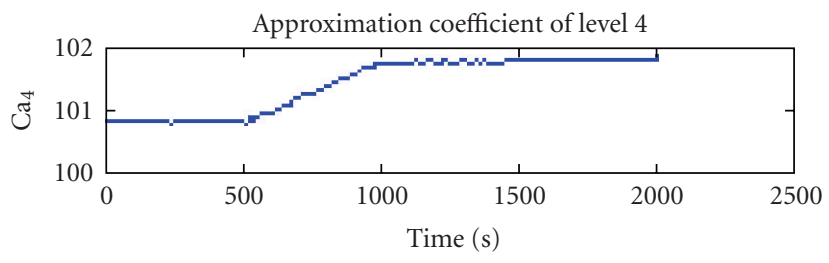

(e)

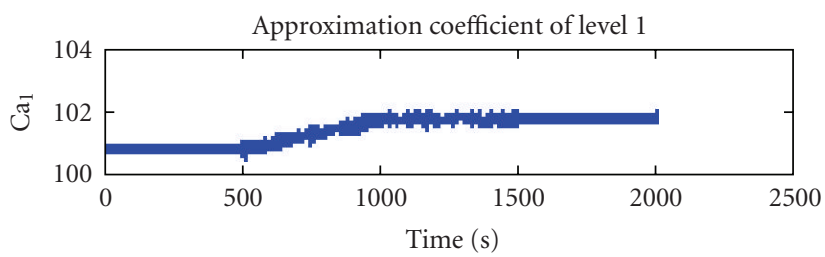

(b)

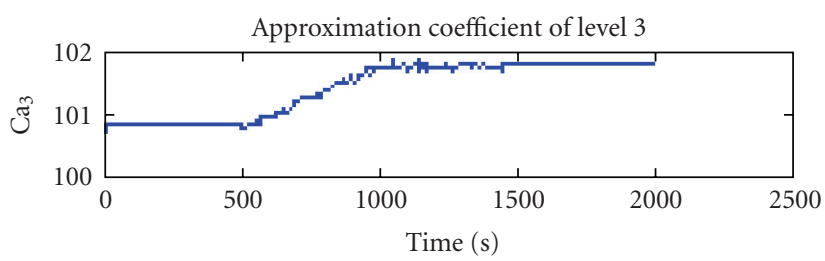

(d)

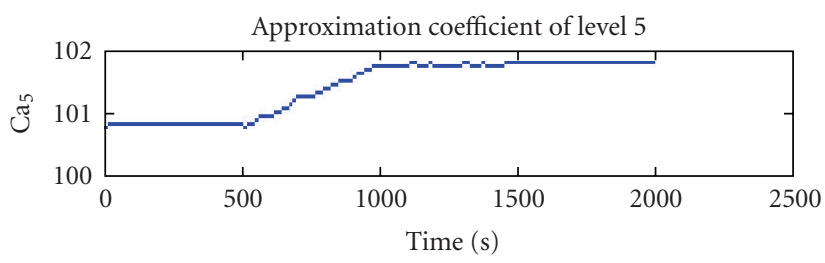

(f)

FIGURE 11: On-line variation trend analysis of the SALS signal by wavelet analysis (wavelet, "sym6," level 5). 


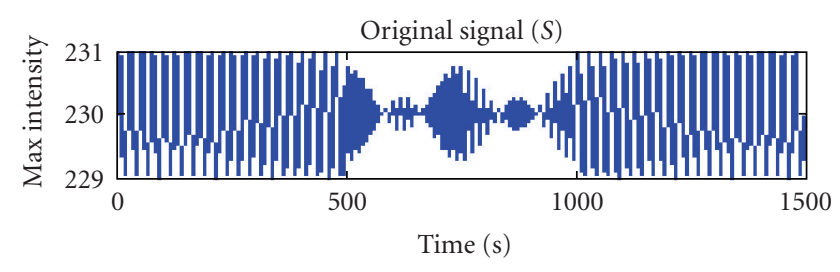

(a)

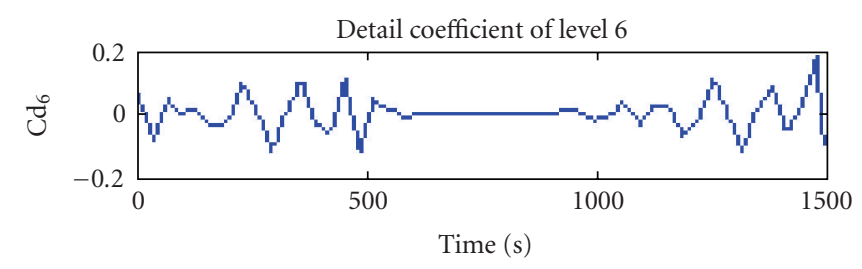

(c)

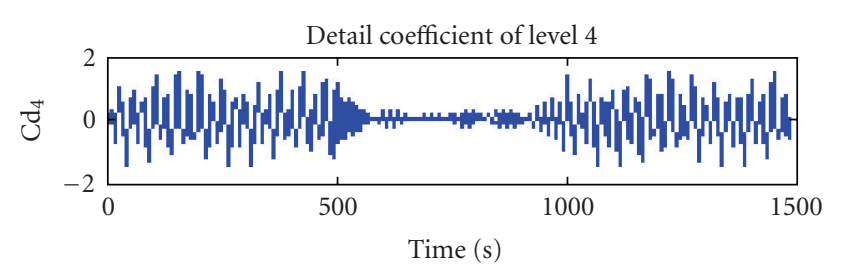

(e)

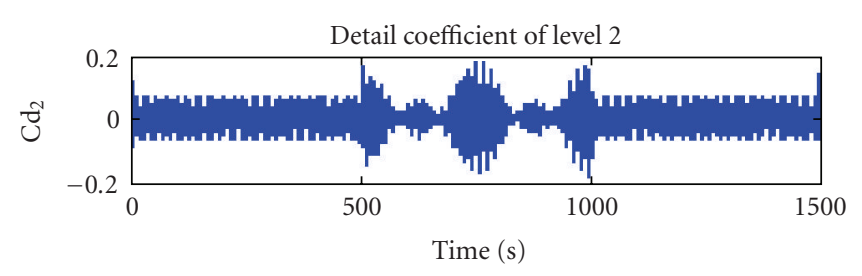

(g)

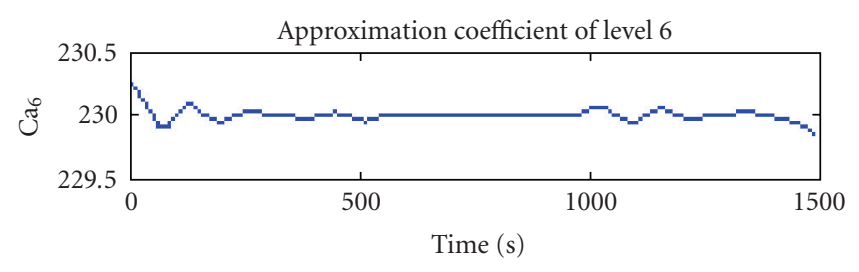

(b)

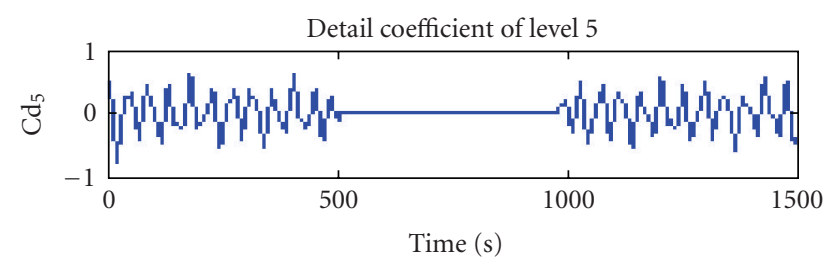

(d)

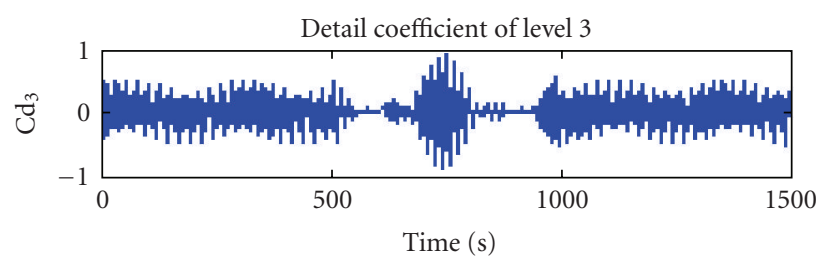

(f)

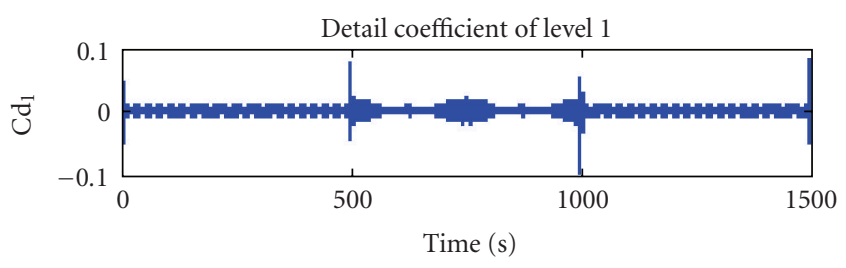

(h)

FIGURE 12: On-line wavelet analysis for the exceptional SALS signal (wavelet, "sym6," level 6).

signal. As shown in Figure 11(a), the variation trend of the original intensity signal $(S)$ is not clear. After multirevolution analysis by wavelet "sym6," the variation trend of the intensity is obvious as shown in Figure 11(f). As shown in Figures $11(\mathrm{~b})-11(\mathrm{f}), \mathrm{Ca}_{j}(j=1,2,3,4,5)$ are the wavelet approximation coefficients of levels $1-5$. It is illustrated that the orientation of molecular chain increases because light intensity is proportional to orientation of molecular chain.

\subsubsection{On-line wavelet analysis for the exceptional SALS signal detection}

The wavelet transform is used to purify the original SALS signal and diagnose the exceptional signal. Figure 12(a) shows the original exceptional maximum intensity signal. Figure 12(b) is the approximation coefficient of level 6. Figures $12(\mathrm{c})-12(\mathrm{~h})$ are the detail coefficients of levels 16 . These coefficients can be feature parameters of the inner structural information of the transparent film for further analysis. As shown in Figure 12(h), the exceptional signal takes place at 500-1000 seconds. The result shows that this technique provides a new tool for diagnosis of exceptional SALS signal.

\section{CONCLUSIONS}

We presented a new technique that can be used to characterize the structural information of transparent film on-line and nonintrusively while the material is processing with different conditions. The technique is based on optical SALS image real-time analysis software and wavelet analysis. Visualization is performed via high-performance analysis software which allows real-time data acquisition and processing the SALS signal.

It is shown that the proposed technique is easy to implement and provides more flexibility approximating the nonlinear relation between the maximum intensity signal and the corresponding vibration intensity (frequency or amplitude). Applying this method to characterize the flow field of polymer melts will be of great interest, since it will contribute information on optical prosperities that have been proven to be useful for obtaining deep insights into 
the molecular and structural parameters of polymers. In our experiments with the increase of vibration intensity, the light intensity matrix becomes stronger and the maximum intensity projection area becomes larger. Because the light intensity is proportional to orientation of molecular chain, it is illustrated that the orientation of molecular chain increases.

In conclusion, this technique is believed to be important and promising to on-line characterize the structural information of transparent film in the multiple-scattering regime.

\section{ACKNOWLEDGMENTS}

The authors acknowledge the supported of the South China Normal University and South China University of Technology. This work was supported by Guangdong Natural Science Fund for free application in 2008: network grid computing task schedule and resource dynamic management research based on P2P strategy, under Project no. 8151063101000040.

\section{REFERENCES}

[1] H. Wang, M. C. Newstein, M. Y. Chang, N. P. Balsara, and B. A. Garetz, "Birefringence and depolarized light scattering of an ordered block copolymer melt under shear flow," Macromolecules, vol. 33, no. 10, pp. 3719-3730, 2000.

[2] Y. A. Akpalu and Y. Lin, "Multivariable structural characterization of semicrystalline polymer blends by small-angle light scattering," Journal of Polymer Science Part B, vol. 40, no. 23, pp. 2714-2727, 2002.

[3] S.-Y. Kwak, "In situ, quantitative characterization of gelation and fusion mechanism in poly(vinyl chloride) plastisols by small angle light scattering (SALS)," Polymer Engineering and Science, vol. 35, no. 13, pp. 1106-1112, 1995.

[4] U. Sundararaj and C. W. Macosko, "Drop breakup and coalescence in polymer blends: the effects of concentration and compatibilization," Macromolecules, vol. 28, no. 8, pp. 2647-2657, 1995.

[5] C. W. Macosko, P. Guégan, A. K. Khandpur, A. Nakayama, P. Marechal, and T. Inoue, "Compatibilizers for melt blending: premade block copolymers," Macromolecules, vol. 29, no. 17, pp. 5590-5598, 1996.

[6] N. C. Beck Tan, S.-K. Tai, and R. M. Briber, "Morphology control and interfacial reinforcement in reactive polystyrene/amorphous polyamide blends," Polymer, vol. 37, no. 16, pp. 3509-3519, 1996.

[7] T. Kyu, M. Mustafa, J. C. Yang, J. Y. Kim, and P. PalffyMuhoray, "Polymerization and thermal quench induced phase separation in polymer dispersed nematic liquid crystals," in Polymer Solutions, Blends and Interfaces, I. Noda and D. N. Rubingh, Eds., pp. 245-271, Elsevier, Amsterdam, The Netherlands, 1992.

[8] B. S. Kim, T. Chiba, and T. Inoue, "Phase separation and apparent phase dissolution during cure process of thermoset/thermoplastic blend," Polymer, vol. 36, no. 1, pp. 67-71, 1995.

[9] M. Okada, K. Fujimoto, and T. Nose, "Phase separation induced by polymerization of 2-chlorostyrene in a polystyrene/dibutyl phthalate mixture," Macromolecules, vol. 28, no. 6, pp. 1795-1800, 1995.

[10] J. Maugey, T. Budtova, and P. Navard, "A light scattering study of phase separation in polymer dispersed liquid crystal composites," in The Wiley Polymer Networks Group Review, Volume 1, Chemical and Physical Networks: Formation and Control of Properties, K. te Nijenhuis and W. Mijs, Eds., pp. 411-420, John Wiley \& Sons, New York, NY, USA, 1998.

[11] K. Sondergaard and J. Lyngaae-Jorgensen, Rheo-Physics of Multiphase Polymer Systems, Technomic, Lancaster, Pa, USA, 1995.

[12] C. Börschig, B. Fries, W. Gronski, C. Weis, and Ch. Friedrich, "Shear-induced coalescence in polymer blends-simulations and rheo small angle light scattering," Polymer, vol. 41, no. 8, pp. 3029-3035, 2000.

[13] Z.-Y. Wang, M. Konno, and S. Saito, "Application of digital image analysis to the characterization of phase-separated structures in polymer blend," Journal of Chemical Engineering of Japan, vol. 24, no. 2, pp. 256-258, 1991.

[14] D. K. Das-Gupta, "Optical and dielectric behaviour of polyethylene," in Proceedings of the IEEE International Symposium on Electrical Insulation (ELINSL '94), pp. 1-11, Pittsburgh, Pa, USA, June 1994.

[15] E. L. Meyer, G. G. Fuller, and R. H. Reamey, "Structure and dynamics of liquid crystalline droplets suspended in polymer liquids," in Liquid Crystal Materials, Devices, and Applications III, vol. 2175 of Proceedings of SPIE, pp. 71-78, San Jose, Calif, USA, February 1994.

[16] J. Maugey, T. Van Nuland, and P. Navard, "Small angle light scattering investigation of polymerisation induced phase separation mechanisms," Polymer, vol. 42, no. 9, pp. 43534366, 2001.

[17] K. Okuyama, Y. Bin, and M. Matsuo, "Polarized small angle light scattering from crystalline polymer gels," Polymer Journal, vol. 55, no. 1, preprint, 55th SPSJ Annual Meeting, p. 742, 2006.

[18] M. K. Endoh, M. Takenaka, T. Inoue, H. Watanabe, and T. Hashimoto, "Shear small-angle light scattering studies of shear-induced concentration fluctuations and steady state viscoelastic properties," Journal of Chemical Physics, vol. 128, no. 16, Article ID 164911, 12 pages, 2008.

[19] Y. A. Akpalu and P. Peng, "Probing the melt miscibility of a commercial polyethylene blend by small-angle light scattering," Materials and Manufacturing Processes, vol. 23, no. 3, pp. 269-276, 2008.

[20] M. Zhang, F. Sun, H. Song, et al., "Design of the quantitative analysis software system for myocardial contrast echocardiography," in Third International Symposium on Multispectral Image Processing and Pattern Recognition, vol. 5286 of Proceedings of SPIE, no. 2, pp. 747-752, Beijing, China, October 2003.

[21] S. J. Sternberg, "CCD advances simplify scientific camera design,” Laser Focus World, vol. 32, no. 1, pp. 101-108, 1996.

[22] D. Lu, Q. Chen, and G. Gu, "High resolution X-ray medical sequential image acquisition and processing system based on PCI interface," in Applications of Digital Image Processing XXVI, vol. 5203 of Proceedings of SPIE, pp. 683-690, San Diego, Calif, USA, August 2003.

[23] G. Bartolacci, P. Pelletier Jr., J. Tessier Jr., C. Duchesne, P.-A. Bossé, and J. Fournier, "Application of numerical image analysis to process diagnosis and physical parameter measurement in mineral processes-part I: flotation control based on froth textural characteristics," Minerals Engineering, vol. 19, no. 6-8, pp. 734-747, 2005, Centenary of Flotation Symposium.

[24] J. J. Liu and J. F. MacGregor, "Estimation and monitoring of product aesthetics: application to manufacturing of 'engineered stone' countertops," Machine Vision and Applications, vol. 16, no. 6, pp. 374-383, 2006. 
[25] S. Lambert, S. Moustier, Ph. Dussouillez, et al., "Analysis of the structure of very large bacterial aggregates by small-angle multiple light scattering and confocal image analysis," Journal of Colloid and Interface Science, vol. 262, no. 2, pp. 384-390, 2003.

[26] A. E. Ismail, G. C. Rutledge, and G. Stephanopoulos, "Using wavelet transforms for multiresolution materials modeling," Computers \& Chemical Engineering, vol. 29, no. 4, pp. 689$700,2005$.

[27] R. Chona, C. S. Suh, and G. A. Rabroker, "Characterizing defects in multi-layer materials using guided ultrasonic waves," Optics and Lasers in Engineering, vol. 40, no. 4, pp. 371-378, 2003.

[28] G. Xian and Z. Wang, "An effective technique of wavelet transform for optical signal real-time processing," in Proceedings of the International Conference on Communications, Circuits and Systems (ICCCAS '05), vol. 1, pp. 653-657, Hong Kong, May 2005.

[29] W. T. Culberson and M. R. Tant, "Device for study of polymer crystallization kinetics via real-time image analysis of small angle light scattering," Journal of Applied Polymer Science, vol. 47, no. 3, pp. 395-405, 1993.

[30] Y. Song and W.-B. Zhang, "Laws of laser speckle movement and their application in flow visualization," in Optical Diagnostics for Fluids/Heat/Combustion and Photomechanics for Solids, vol. 3783 of Proceedings of SPIE, pp. 89-100, Denver, Colo, USA, July 1999.

[31] Y. Song, R. Kulenovic, M. Groll, and Z. Guo, "Effects of speckle displacement on speckle interferometry for measurement of phase object," Optics Communications, vol. 139, no. 1-3, pp. 24-30, 1997.

[32] C. Batur, M. H. Vhora, M. Cakmak, and T. Serhatkulu, "Online crystallinity measurement using laser Raman spectrometer and neural network," ISA Transactions, vol. 38, no. 2, pp. 139-148, 1999.

[33] J. Zhou and J. Sheng, "Small angle light backscattering of polymer blends: 1. Multiple scattering," Polymer, vol. 38, no. 15, pp. 3727-3731, 1997.

[34] Q. Yang, Study on phase behavior of PS/SAN blends at static state and under shear flow with SALS method, Ph.D dissertation, Sichuan University, Sichuan, China, 2002.

[35] I. Daubechies, "The wavelet transform, time-frequency localization and signal analysis," IEEE Transactions on Information Theory, vol. 36, no. 5, pp. 961-1005, 1990.

[36] I. Daubechies, "Orthogonal bases of compactly supported wavelets," Communications on Pure and Applied Mathematics, vol. 41, no. 7, pp. 909-996, 1988.

[37] S. Mallat, "A theory of multi-resolution signal decomposition: the wavelet representation," IEEE Transactions on Pattern Analysis and Machine Intelligence, vol. 11, no. 7, pp. 674-693, 1989.

[38] G. Evangelista, "Orthogonal wavelet transforms and filter banks," in Proceedings of the 6th Multidimensional Signal Processing Workshop (MDSP '89), p. 100, Pacific Grove, Calif, USA, September 1989.

[39] N. H. Abu-Zahra and A. Seth, "In-process density control of extruded foam PVC using wavelet packet analysis of ultrasound waves," Mechatronics, vol. 12, no. 9-10, pp. 10831095, 2002.

[40] D. D. Rancic, P. M. Eferica, S. J. Djordjevic-Kajan, A. T. Kostic, M. S. Smiljanic, and P. D. Vukovic, "Digital signal and image processing for meteorological radars," in Proceedings of the 10th Mediterranean Electrotechnical Conference (MALECON '00), vol. 2, pp. 623-626, Lemesos, Cyprus, May 2000. 


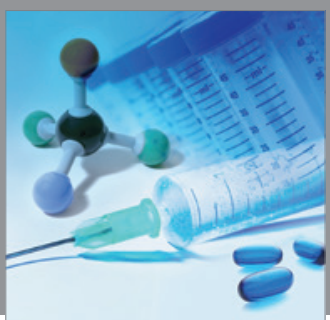

International Journal of

Medicinal Chemistry

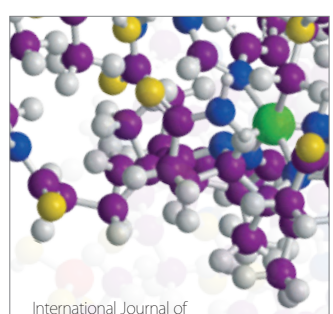

Carbohydrate Chemistry

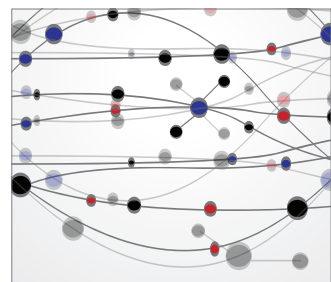

The Scientific World Journal
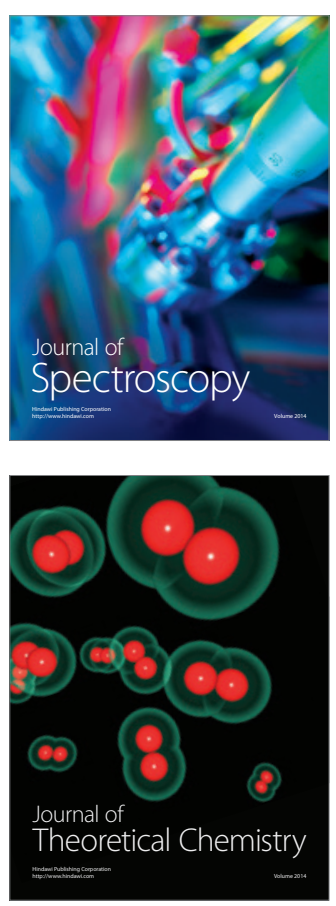
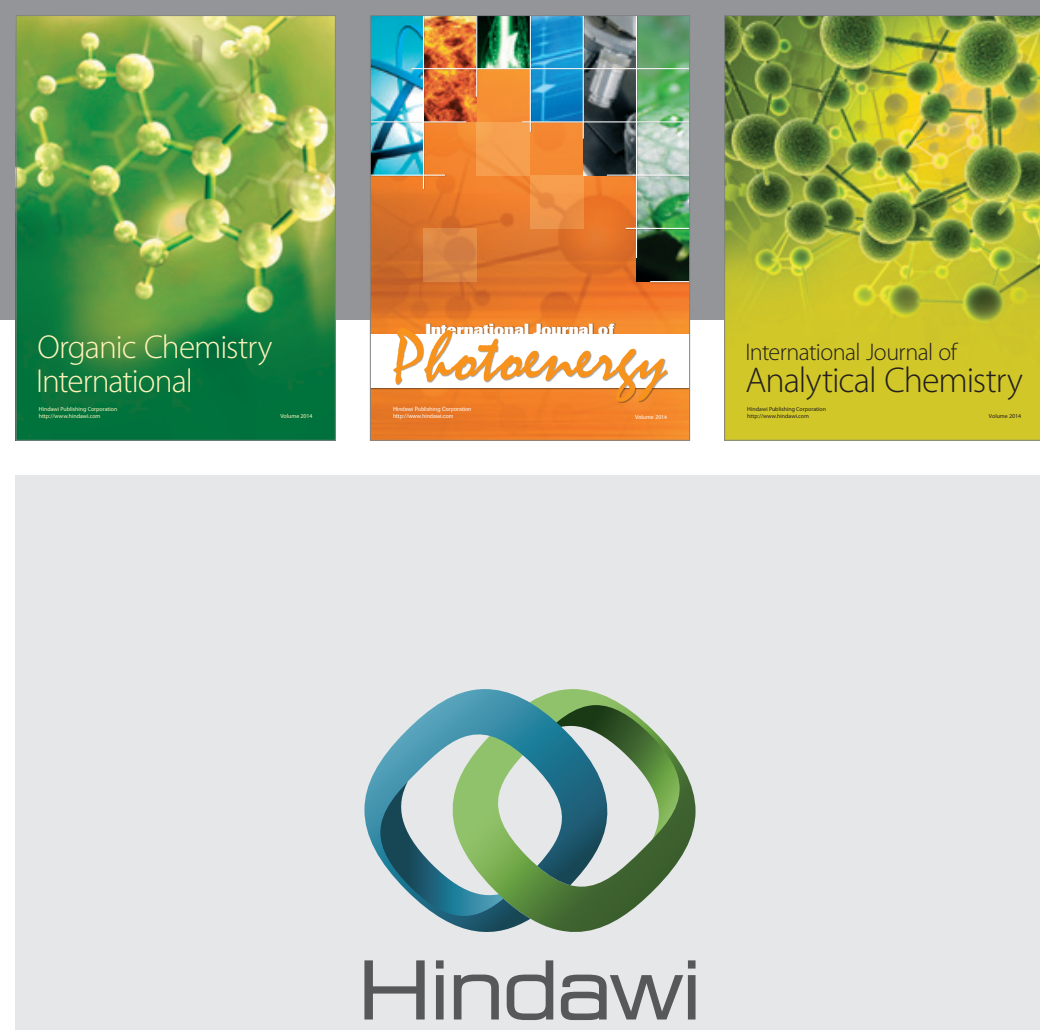

Submit your manuscripts at

http://www.hindawi.com
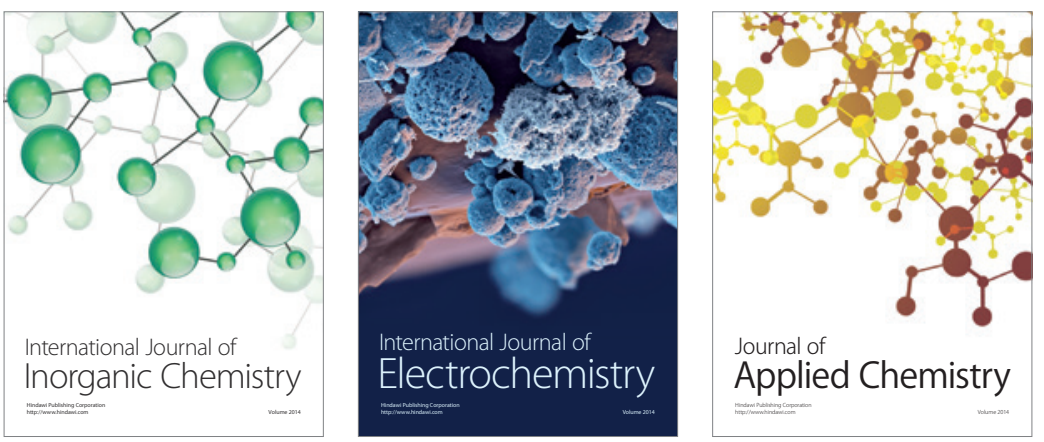

Journal of

Applied Chemistry
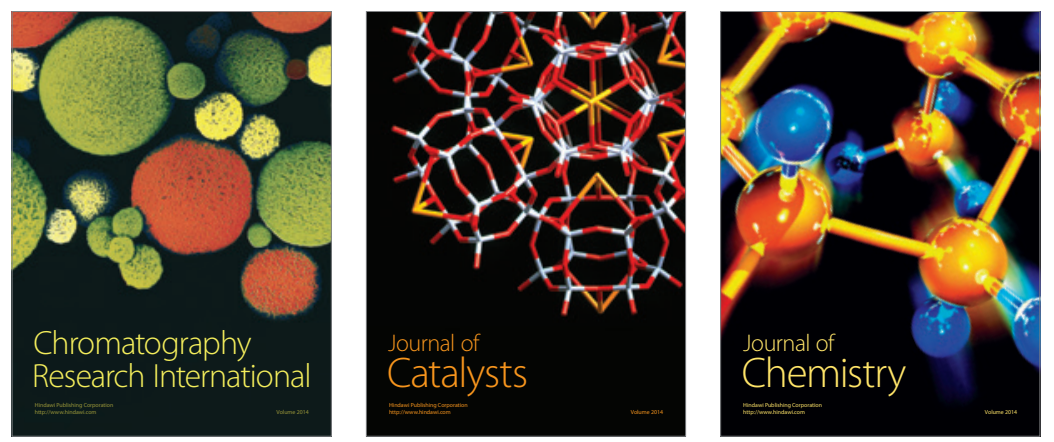
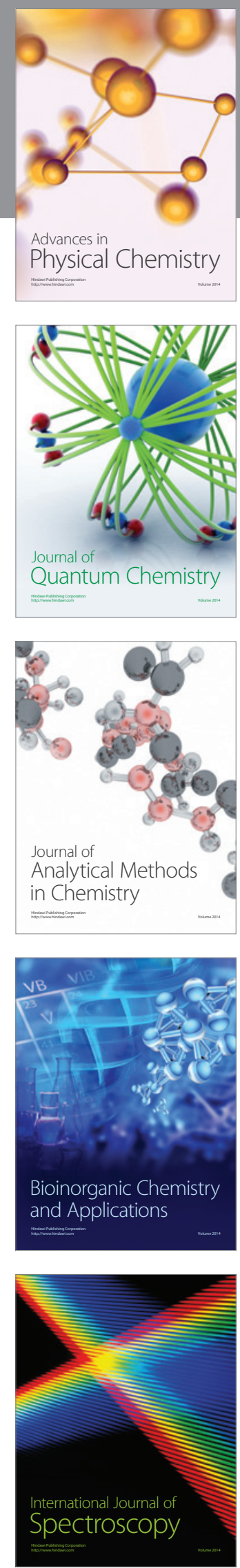